\title{
Comparative Genome Analysis of Psychrobacillus Strain PB01, Isolated from an Iceberg $\mathbb{S}$
}

\author{
Jun Young Choi ${ }^{1}$, Sun Chang Kim ${ }^{2}$, and Pyung Cheon Lee ${ }^{1 *}$ \\ ${ }^{1}$ Department of Molecular Science and Technology and Department of Applied Chemistry and Biological Engineering, Ajou University, \\ Suwon 16499, Republic of Korea \\ ${ }^{2}$ Department of Biological Sciences, Korea Advanced Institute of Science and Technology, Daejeon 34141, Republic of Korea
}

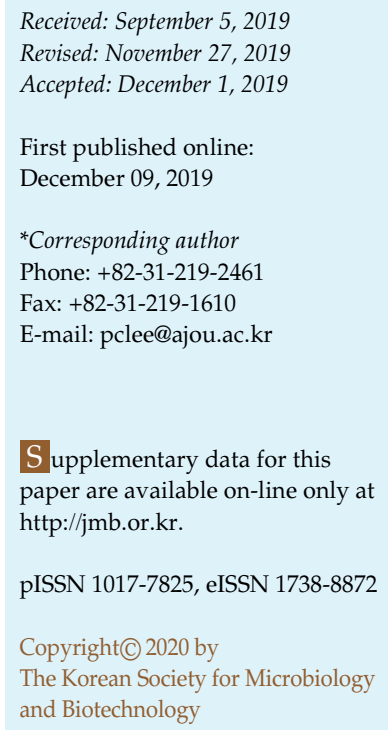

S upplementary data for this paper are available on-line only at http://jmb.or.kr.

pISSN 1017-7825, eISSN 1738-8872

Copyright@ 2020 by

The Korean Society for Microbiology and Biotechnology

A novel psychrotolerant Psychrobacillus strain PB01, isolated from an Antarctic iceberg, was comparatively analyzed with five related strains. The complete genome of strain PB01 consists of a single circular chromosome $(4.3 \mathrm{Mb})$ and a plasmid $(19 \mathrm{~Kb})$. As potential low-temperature adaptation strategies, strain PB01 has four genes encoding cold-shock proteins, two genes encoding DEAD-box RNA helicases, and eight genes encoding transporters for glycine betaine, which can serve as a cryoprotectant, on the genome. The pan-genome structure of the six Psychrobacillus strains suggests that strain PB01 might have evolved to adapt to extreme environments by changing its genome content to gain higher capacity for DNA repair, translation, and membrane transport. Notably, strain PB01 possesses a complete TCA cycle consisting of eight enzymes as well as three additional Helicobacter pylori-type enzymes: ferredoxin-dependent 2-oxoglutarate synthase, succinyl-CoA/acetoacetyl-CoA transferase, and malate/quinone oxidoreductase. The co-existence of the genes for TCA cycle enzymes has also been identified in the other five Psychrobacillus strains.

Keywords: Psychrobacillus, iceberg, TCA cycle, plasmid

\section{Introduction}

Cold-adapted bacteria are classified into psychrophiles, which can grow at $0-20^{\circ} \mathrm{C}$, or psychrotolerants (or psychrotrophs), which can grow at up to $\sim 30^{\circ} \mathrm{C}$ [1]. Besides their biodiversity and ecological significance, cold-adapted bacteria have increasingly attracted attention due to their potential for biotechnological application, particularly in the use of cold-active enzymes and biomaterials in the food and chemical industries. A psychrotolerant strain, strain PB01, was isolated from an Antarctic iceberg near King Sejong Station. This strain is a pale yellow-colored, grampositive rod-shaped bacterium belonging to the family Bacillaceae [2]. Three Bacillus strains were reclassified under the genus Psychrobacillus, based on polyphasic reexamination [2]. Currently, five strains of Psychrobacillus have been reported: P. psychrotolerans DSM $11706^{\mathrm{T}}$, P. psychrodurans DSM $11713^{\mathrm{T}}, P$. insolitus DSM $5^{\mathrm{T}}, P$. soli $\mathrm{NHI}-2^{\mathrm{T}}$, and $P$. lasiicapitis NEAU-3TGS17 ${ }^{\mathrm{T}}$ [3-6].

Moreover, the genomes of only five Psychrobacillus strains, namely P. psychrotolerans, P. psychrodurans DSM 11713, Psychrobacillus sp. FJAT-21963, Psychrobacillus sp. OK032, and Psychrobacillus sp. OK028, have been sequenced and deposited in public databases. However, the five genomes are not completely sequenced and thus do not contain sufficient data to provide the full genomic information of Psychrobacillus strains. The genome content and metabolic pathways of a Psychrobacillus strain whose genome is completely sequenced may provide a model strain for utilization in basic and biotechnological research. Furthermore, the genome-guided discovery of mobilomes, including plasmids, can accelerate the development of genetic engineering tools useful for manipulating Psychrobacillus strains. So far, however, there has been no report of a plasmid present in the five type strains of genus Psychrobacillus.

Here, we report the genomic characterization of the novel psychrotolerant strain PB01, which was isolated from an Antarctic iceberg, and provide a complete genome sequence of strain PB01. A comparative analysis of genomes of the five strains of Psychrobacillus was also described. 


\section{Materials and Methods}

\section{Genome Sequencing and Annotation}

The genomic DNA of strain PB01 was extracted using the Genomic DNA Kit (Macrogen, Korea). The genome of strain PB01 was sequenced in SMRT cells using the Pacific Biosciences RS II single-molecule real-time sequencing technology kit (Pacific Biosciences, USA). Following sub-read filtering of raw read data via the PacBio RS II sequencer, 186,405 long reads and 1,658,531,999 base pairs, with a 386-fold genome coverage, were generated and de novo assembled using the Canu v1.3 assembler [7]. The circularization of contigs was performed using Circlator [8]. The open reading frames (ORFs) of one chromosome and one plasmid were predicted using the RAST server online [9]. tRNA and rRNA were predicted using tRNAscan-SE v1.21 [10], and RNAmmer v1.2 [11], respectively. Function predictions of the ORFs of the chromosome were made based on RPS-BLAST and BLASTp searches (e-value $<10^{-3}$ ) against the non-redundant GenBank protein database (www.ncbi.nlm.nih.gov/protein), the clusters of orthologous groups (COG) database (www.ncbi.nlm.nih.gov/ COG), and the KEGG database (www.genome.jp/kegg). Graphical circular maps of the chromosome and the plasmid were produced using Circos v0.67 [12].

\section{Analysis of Average Nucleotide Identity (ANI)}

The reference draft genome sequences of $P$. psychrotolerans (NZ_FOXU00000000.1), P. psychrodurans DSM 11713 (NZ_FOUN 00000000.1), Psychrobacillus sp. FJAT-21963 (NZ_LJIY00000000.1), Psychrobacillus sp. OK032 (NZ_FOGY00000000.1), and Psychrobacillus sp. OK028 (NZ_FNHY00000000.1) were downloaded from NCBI (www.ncbi.nlm.nih.gov/refseq). The draft genome contigs of the five strains were realigned against the complete genome of strain PB01 using Mauve Contig Mover [13]. The average nucleotide identity (ANI) values of strain PB01 and the five Psychrobacillus strains were analyzed using pyani v0.2.7 [14], a Python module, with default parameters. The synteny blocks (locally collinear blocks) of strain PB01 and the five Psychrobacillus strains were analyzed using progressiveMauve v.2.3.1 [15].

\section{Pan-Genome and Metabolic Pathway Analysis of Psychrobacillus Strains}

The annotation files (GFF3) of strain PB01 and the five Psychrobacillus strains were generated using Prokka-1.12 [16] and were used for pan-genome analysis using Roary v3.11.2 [17] with a 70\% minimum BLASTp percentage identity. The output files of Roary v3.11.2 were used to analyze and visualize the core and accessory genomes of Psychrobacillus strains using R (https:// www.r-project.org/). Gene differences between strain PB01 and the five Psychrobacillus strains were visualized using the UpsetR package [18]. COG assignment of the core and unique genes of the six Psychrobacillus strains was carried out as described in the "Genome sequencing and annotation" section and were presented as a heatmap using R (v. 3.4.1). The TCA cycle, glyoxylate and anaplerotic pathways of Psychrobacillus sp. PB01 were in silico analyzed using the Pathway Tools v20.5 [19]. Phylogenetic trees were reconstructed using the neighbor-joining method [20].

\section{Results and Discussion}

\section{Genome Analysis of Strain PB01}

The genome of strain PB01 consists of a 4,332,095-bp circular chromosome with a G+C content of $36.0 \%$ (Fig. S1A) and one extrachromosomal circular plasmid $(19,243 \mathrm{bp})$ with a G+C content of $34.3 \%$ (Fig. S1B). A total of 4,377 coding DNA sequences (CDSs) of the chromosome were predicted along with 33 rRNA and 77 tRNA genes (Table 1), resulting in a gene density of 1,036 genes/megabase. A total of 28 CDSs of the plasmid named 'pPB01' were predicted with a gene density of 1.56 genes/kilobase (Table 1 ). The identified 2,757 CDSs of the chromosome were classified into functional categories based on clusters of orthologous genes (COG) designation [21]. The classified genes are presented in the circular maps with color codes (Figs. S1A and S1B). The most abundant COG category of chromosome, except for [S] Function unknown (304 CDSs, 10.1\%), was [R] General function prediction only (384 CDSs, 12.8\%), followed by [E] Amino acid transport and metabolism (313 CDSs, 10.4\%), [K] Transcription (227 CDSs, 7.6\%), and [L] Replication, recombination and repair (223 CDSs, $7.4 \%$ ), accounting for $38.1 \%$ of the overall CDS $(1,147 / 3,007$ CDSs) (Table S1). Generally, cold environments place physicochemical stresses, such as low water activity, excessive UV or radiation, low solute diffusion, and low nutrient availability, on psychrophiles/psychrotolerants [1, 22]. To cope with unfavorable environments psychrophiles/ psychrotolerants have evolved adaptive mechanisms by changing their genome content to gain high capacity for DNA repair, translation, and membrane transport [22]. Therefore, the high abundance of the COG categories might play important roles in the adaptation of strain PB01 to unique geographical locations (iceberg) [23].

Table 1. General genome features of Psychrobacillus strain PB01.

\begin{tabular}{lccc}
\hline \multicolumn{1}{c}{ Attribute } & Chromosome & Plasmid & Total \\
\hline Genome size (bp) & $4,332,095$ & 19,243 & $4,351,338$ \\
Number of contigs & 1 & 1 & 2 \\
G+C content (\%) & 36.0 & 34.3 & 36 \\
CDS & 4,377 & 28 & 4,405 \\
rRNA (5S, 16S, 23S) & 33 & 0 & 33 \\
tRNA & 77 & 0 & 77 \\
\hline
\end{tabular}




\section{Comparative Genome Analysis of Psychrobacillus Strains}

The general chromosomal features of strain PB01 were compared to the published chromosomes of the five Psychrobacillus strains (Table S2). The genomic G+C content of all six Psychrobacillus strains was $35.8-37.0 \%$, which is within the reported range for Psychrobacillus strains. The chromosome sizes of all six Psychrobacillus strains were between 3.61 and $4.39 \mathrm{Mb}$, suggesting extensive gene gain and loss in the genus during evolutionary events. High variations in genome size were also observed in some psychrophile Psychrobacter strains [24]. Notably, the chromosome of strain PB01 encodes a higher number of copies of rRNA and tRNA genes (33 and 77) than any of the other five strains (Table S2). A high level of redundant rRNA and tRNA genes on genomes of psychrophiles is one of the survival strategies at low temperatures [25]. As such, the accelerated translation of special proteins caused by multiple copies of rRNA and tRNA genes may facilitate the fast cellular responses of strain PB01 to environmental changes, including rapid temperature shifts [26-28].

Average nucleotide identity (ANI) analysis helps elucidate the relationship between two strains via the identity/ similarity values of the homologous regions of two genomes [29]. Thus, to obtain information on the lineage of strain PB01, compared to that of the five related strains, ANI analysis was performed using pyani with three alignment algorithms: a mummer (ANIm), blastn (ANIb), and blastall (ANIblastall), and alignment-free algorithm tetranucleotide signature frequencies (TETRA) (https:// github.com/widdowquinn/pyani). The ANI values of the strain PB01 chromosome, compared against the genomes of the five related strains, were $0.836-0.866$ with ANIm, 0.763-0.801 with ANIb, 0.758-0.795 with ANIblastall, and 0.982-0.991 with TETRA (Fig. S3), suggesting strain PB01 as a new species, in the genus Psychrobacillus, based on the commonly used ANIm, ANIb, and ANIblastall threshold values $(<0.95-0.96)$ [29], and the TETRA threshold value $(<0.99)$ for species delineation [30].

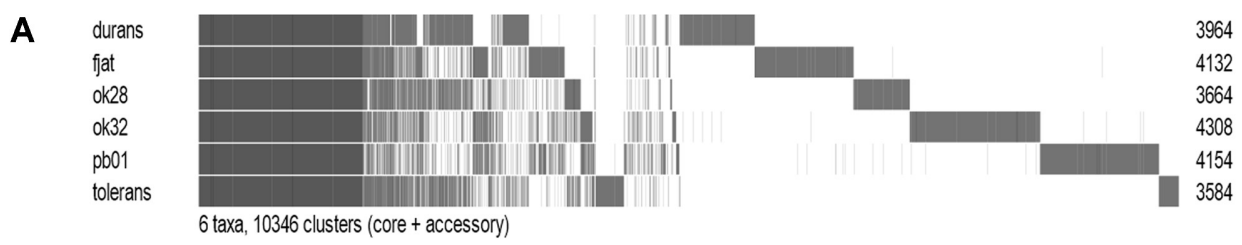

B

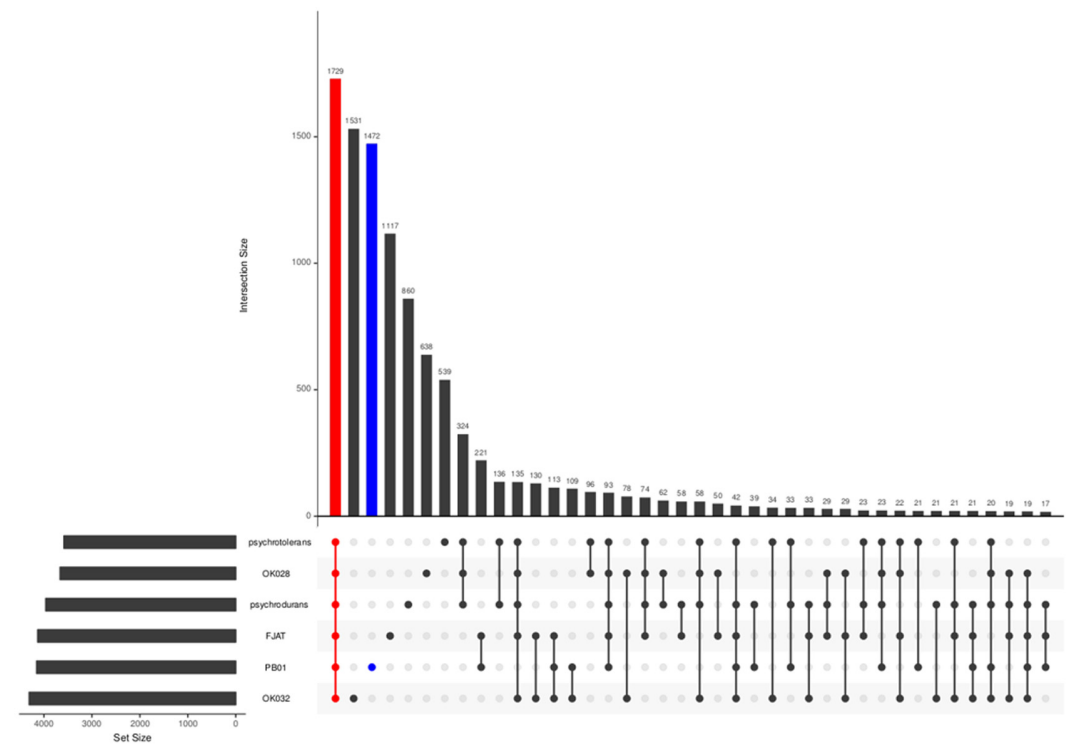

Fig. 1. Pan-genome analysis of Psychrobacillus sp. PB01 and five related Psychrobacillus strains.

(A) A gene presence/absence matrix plot generated using Roary. The numbers on the right represent the total number of core and unique genes of each Psychrobacillus strain. (B) Gene sharing among the Psychrobacillus sp. PB01 and the five other representative strains of Psychrobacillus. The bars represent the number (or size) of each intersection (dark circles) shown in the combination matrix. A red bar represents the number of core genes of the six Psychrobacillus strains; a blue bar highlights the number of unique genes of Psychrobacillus sp. PB01. The plot was drawn using UpSetR. Durans, P. psychrodurans DSM 11713; FJAT, Psychrobacillus sp. FJAT-21963; OK28, Psychrobacillus sp. OK028; OK32, Psychrobacillus sp. OK032; PB01, Psychrobacillus sp. PB01; Tolerans, P. psychrotolerans. 
Following ANI calculation, synteny linkage of strain PB01 against the five related strains was further analyzed using progressiveMauve. As the genomes of the five reference strains were fragmented (Table S2), the contigs of the genomes were first reordered against the complete genome of strain PB01 with Mauve Contig Mover, following which synteny blocks (locally collinear blocks [LCB]) were analyzed using progressiveMauve with the recommended parameters (http://darlinglab.org/mauve/ user-guide/progressivemauve.html). As inferred from the ANI analysis results, several large-scale synteny blocks (or LCB) were identified between the genomes of strain PB01 and the five related strains (Fig. S2A), reflecting a high degree of conservation among Psychrobacillus genomes. Outgoing link size in 5-kb windows and outgoing link number in 5-kb windows were shown in synteny linkages between the genomes of strain PB01 and the five related strains (Fig. S2B).

\section{Pan-Genome Analysis of Psychrobacillus Strains}

The pan-genome consists of the total number of genes of a species and can be used to obtain evolutionary event information for that species, including gene loss and gain [31]. Using Roary with a 70\% minimum BLASTp identity [32], the pan-genomes of strain PB01 and the five Psychrobacillus strains were analyzed to determine the variations in gene content (Fig. 1). The pan-genome structure of the six strains comprises a total of 10,346 genes (Fig. 1A): 1,729 core genes $(99 \%<=$ strains $<=100 \%)$ and 8,617 shell genes $(15 \%<=$ strains $<95 \%)$. There are no soft core $(95 \%$

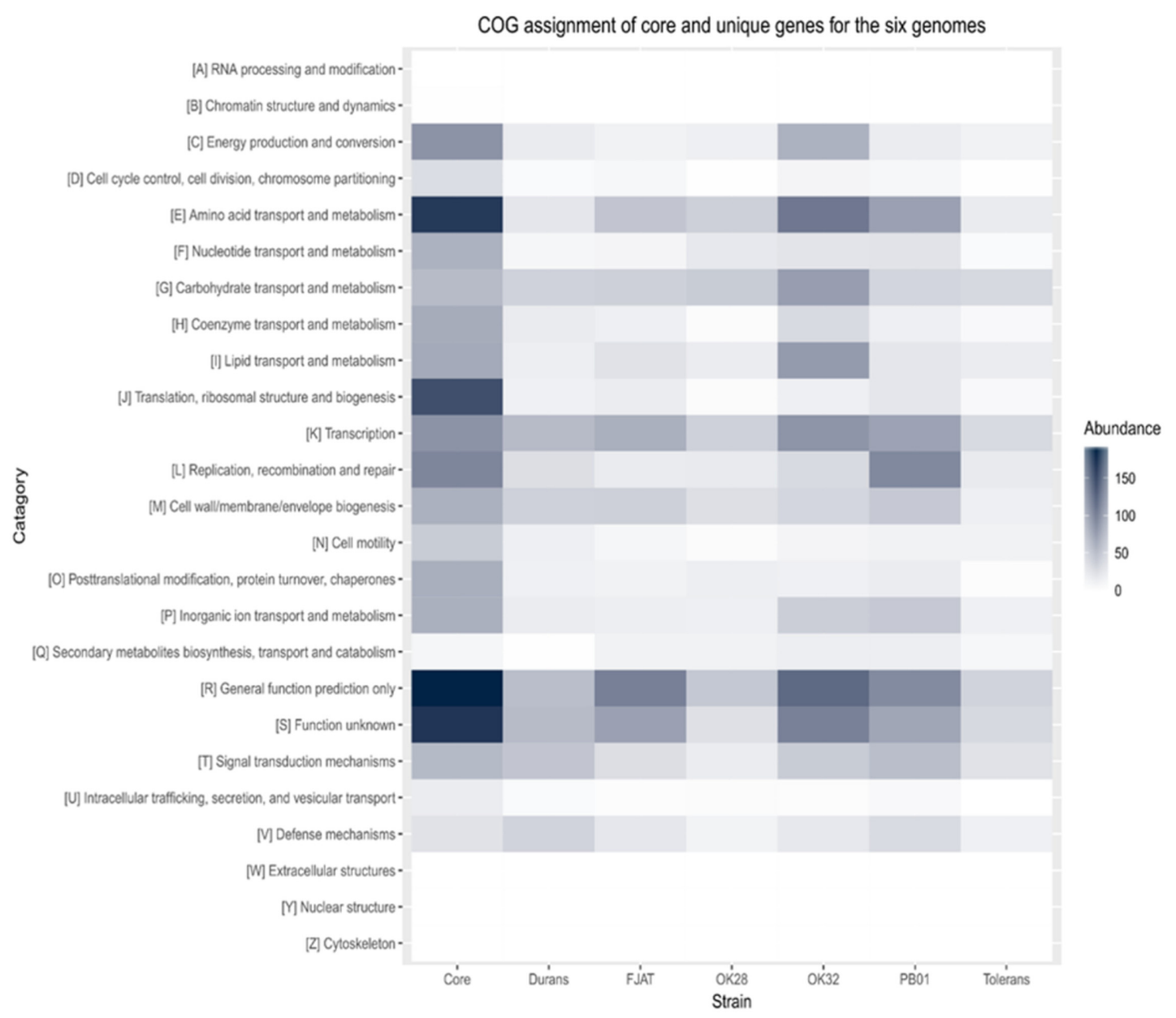

Fig. 2. COG assignment of core and unique genes of the six Psychrobacillus strains.

The comparative COG assignments of the core and unique genes of the six Psychrobacillus strains, obtained using Roary, were presented through a heatmap. Core, the core genes of the six Psychrobacillus strains; Durans, the unique genes of P. psychrodurans DSM 11713; FJAT, the unique genes of Psychrobacillus sp. FJAT-21963; OK28, the unique genes of Psychrobacillus sp. OK028; OK32, the unique genes of Psychrobacillus sp. OK032; PB01, the unique genes of Psychrobacillus sp. PB01; Tolerans, the unique genes of P. psychrotolerans. Scale of abundance (the number of genes) was represented by a continuous color gradient. 
$<=$ strains $<99 \%)$ or cloud $(0 \%<=$ strains $<15 \%)$ genes. The highest number of unique (or accessory) genes present in the Psychrobacillus strains (Fig. 1B) is 1,531 (OK032), followed by 1,472 (PB01), 1,117 (FJAT), 860 (pyschrodurans), 638 (OK028), and 539 (psychrotolerans). Unique genes of a species are usually involved in niche-specific adaptation [32]. The number of unique genes of each strain are related to the genome size of each Psychrobacillus strain (Table S2). The COG assignments of the core and unique genes of the six Psychrobacillus strains, including strain PB01, were

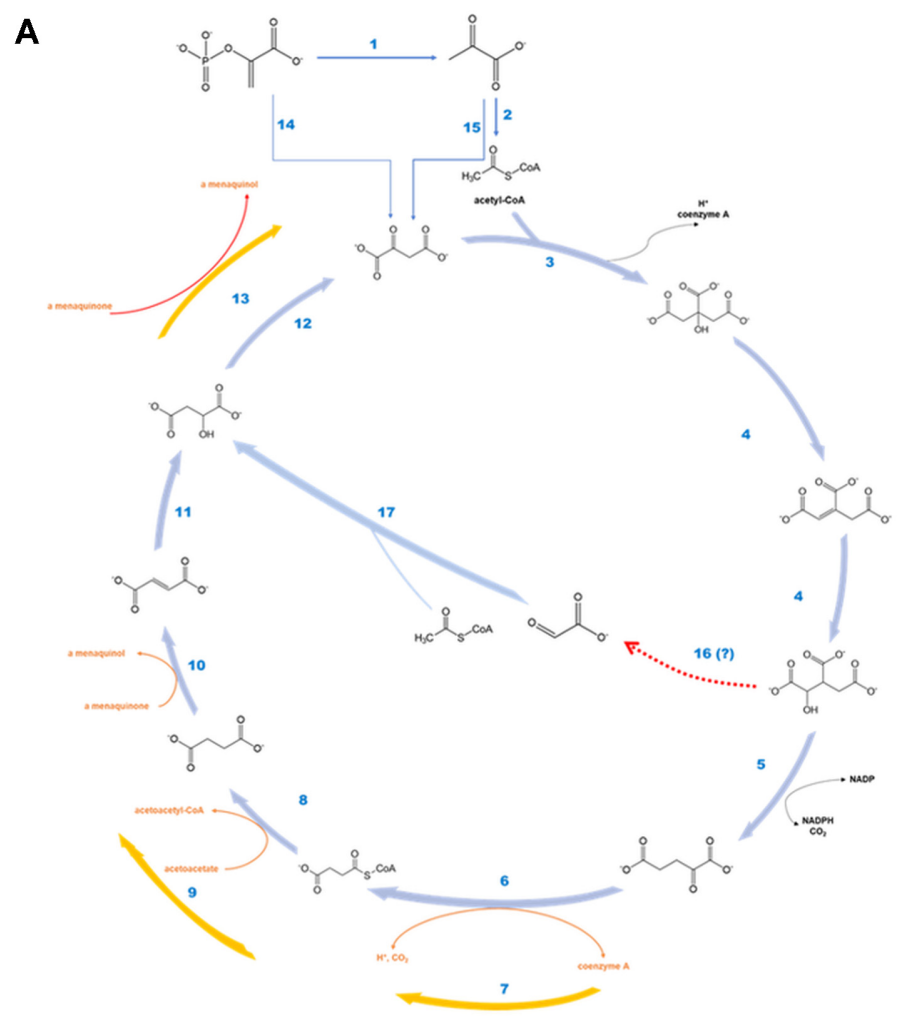

B Ferredoxin-dependent 2-
oxoglutarate oxidoreductase

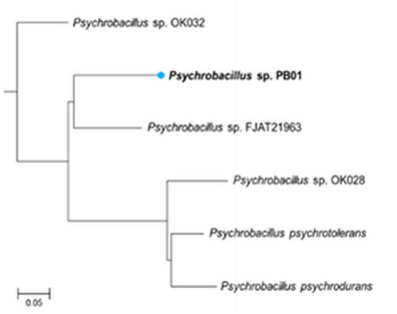

Succinyl-CoA/3-ketoacid-CoA transferase

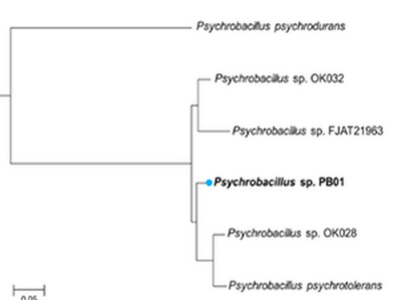

Malate/quinone oxidoreductase

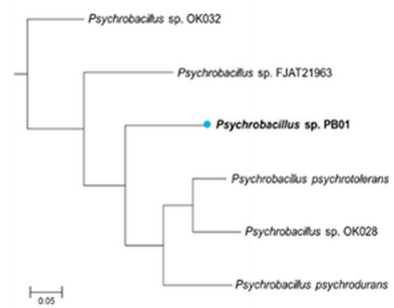

Fig. 3. In silico prediction of TCA cycle, glyoxylate bypass, and anaplerotic pathway of Psychrobacillus sp. PB01 (A) and phylogenetic trees of ferredoxin-dependent 2-oxoglutarate oxidoreductase, succinyl-CoA/3-ketoacid CoA transferase, and malate/quinone oxidoreductase of Psychrobacillus strains (B).

(A) The TCA cycle, glyoxylate and anaplerotic pathways of Psychrobacillus sp. PB01 were in silico analyzed using the Pathway Tools. Additional three reactions $(7,9$, and 13) of the TCA cycle were represented by yellow arrows. The pathway enzymes involved in reactions are (1) Pyruvatekinase, (2) Pyruvate dehydrogenase, (3) Citrate synthase, (4) Aconitase, (5) Isocitrate dehydrogenase, (6) NAD ${ }^{+}$-dependent 2oxoglutarate dehydrogenase, (7) ferredoxin-dependent 2-oxoglutarate oxidoreductase, (8) Succinyl-CoA ligase, (9) Succinyl-CoA/3-ketoacid CoA transferase, (10) Succinate dehydrogenase, (11) Fumarate hydratase, (12) Malate dehydrogenase, (13) Malate/quinone oxidoreductase, (14) Phosphoenolpyruvate carboxykinase, (15) Pyruvate carboxylase, (16) a missing enzyme, and (17) Malate synthase. (B) Phylogenetic trees of ferredoxin-dependent 2-oxoglutarate oxidoreductase (subunit alpha), succinyl-CoA/3-ketoacid CoA transferase (subunit A), and malate/quinone oxidoreductase of six Psychrobacillus strains were obtained using the neighbor-joining method. Bar, 0.05 substitutions per amino acid position. 
analyzed to gain an insight into the difference between the gene functions of the strains (Fig. 2). Among the 1,729 core genes of the six strains, 1,513 genes were assigned into COG categories. The most abundant COG category of the core genes, except for [R] General function prediction only (187 genes, 12.4\%) and [S] Function unknown (168 genes, 11.1\%), was [E] Amino acid transport and metabolism (165 genes, $10.9 \%$ ) and [J] Translation, ribosomal structure and biogenesis (144 genes, 9.5\%). Notably, the unique genes assigned to [L] Replication, recombination and repair (93 genes), [T] Signal transduction mechanisms (50 genes), [P] Inorganic ion transport and metabolism (41 genes), and [J] Translation, ribosomal structure and biogenesis (19 genes) in strain PB01 were more abundant than those of the other five strains. As potential low-temperature adaptation strategies [23] strain PB01 has four genes encoding coldshock proteins, two genes encoding DEAD-box RNA helicases, and eight genes encoding transporters for glycine betaine, which can serve as a cryoprotectant [33], on the genome.

\section{In Silico Analysis of TCA Cycle and Glyoxylate Bypass of Psychrobacillus Strains}

In silico pathway analysis uncovered that the genome of strain PB01 has genes encoding all of the enzymes involved in the TCA cycle (Fig. 3A), suggesting that the TCA cycle of strain PB01 would be fully operative in the oxidative direction [34]. Notably, in addition to the complete TCA cycle enzymes, additional genes encoding three enzymes were annotated as ferredoxin-dependent 2-oxoglutarate synthase, succinyl-CoA/ acetoacetyl-CoA transferase, and malate/quinone oxidoreductase. These three enzymes had been reported as alternative enzymes of the TCA cycle in Helicobacter pylori [35]. It remains a question as to whether two types of the enzymes would be fully active simultaneously and function differently under physiological or environmental conditions. As suggested in the previous study [36], it is possible that two types of enzymes would function differently to meet metabolic needs in the cellular carbon metabolism. Comparative genome analysis revealed that the genomes of the other Psychrobacillus strains also encode the three enzymes (Fig. 3B). Thus, the observed coexistence of TCA cycle enzymes would be unique in Psychrobacillus strains. The anaplerotic formation of oxaloacetate from phosphoenolpyruvate (PEP) and pyruvate was predicted by the presence of genes encoding PEP carboxykinase and pyruvate carboxylase, respectively (Fig. 3A). However, there is no candidate gene encoding PEP carboxylase, which is the third anaplerotic enzyme, in the genome of strain PB01. Among two glyoxylate pathway enzymes (isocitrate lyase and malate synthase) only one gene encoding malate synthase was identified. There are no homologs of isocitrate lyase genes in the genome of strain PB01.

In summary, the complete genome of strain PB01 consists of a single circular chromosome and a plasmid. Comparative genomic analysis suggests extensive gene gain and loss in the genus during evolutionary events. The pan-genome of the six strains analyzed comprises 1,729 core genes and 8,617 unique (accessory) genes. An abundance of unique genes assigned to (i) Replication, recombination, and repair, (ii) Signal transduction mechanisms, (iii) Inorganic ion transport and metabolism, and (iv) Translation, ribosomal structure, and biogenesis in the chromosome of strain PB01 suggests that they play critical roles in the adaptation of this strain to extreme environments. Notably, Psychrobacillus strains possess a complete TCA cycle as well as three additional $H$. pylori-type enzymes. The co-presence of the genes for TCA cycle enzymes could facilitate the research for variation and evolution of the TCA cycle.

\section{Acknowledgments}

This work was supported by the National Research Foundation of Korea (NRF) grant funded by the government of South Korea (grant numbers 2014M3A6A8066439 and 2017R1A2B4011899).

\section{Conflict of Interest}

The authors have no financial conflicts of interest to declare.

\section{References}

1. DasSarma P, DasSarma S. 2018. Survival of microbes in Earth's stratosphere. Curr. Opin. Microbiol. 43: 24-30.

2. Krishnamurthi S, Ruckmani A., Pukall R, Chakrabarti T. 2010. Psychrobacillus gen. nov. and proposal for reclassification of Bacillus insolitus Larkin \& Stokes, 1967, B. psychrotolerans Abd-El Rahman et al., 2002 and B. psychrodurans Abd-El Rahman et al., 2002 as Psychrobacillus insolitus comb. nov., Psychrobacillus psychrotolerans comb. nov. and Psychrobacillus psychrodurans comb. nov. Syst. Appl. Microbiol. 33: 367-373.

3. Abd El-Rahman, HA, Fritze D, Sproer C, Claus D. 2002. Two novel psychrotolerant species, Bacillus psychrotolerans sp. nov. and Bacillus psychrodurans sp. nov., which contain ornithine in their cell walls. Int. J. Syst. Evol. Microbiol. 52: 2127-2133. 
4. Larkin, J. M., Stokes, J. L. 1967. Taxonomy of psychrophilic strains of Bacillus. J. Bacteriol. 94: 889-895.

5. Pham V H, Jeong SW, Kim J. 2015. Psychrobacillus soli sp. nov., capable of degrading oil, isolated from oil-contaminated soil. Int .J. Syst. Evol. Microbiol. 65: 3046-3052.

6. Shen Y, Fu Y, Yu Y, Zhao J, Li J, Li Y, et al. 2017. Psychrobacillus lasiicapitis sp. nov., isolated from the head of an ant (Lasius fuliginosus). Int. J. System. Evol. Microbiol. 67: 4462-4467.

7. Koren S, Walenz BP, Berlin K, Miller JR, Bergman NH, Phillippy AM. 2017. Canu: scalable and accurate long-read assembly via adaptive k-mer weighting and repeat separation. Genome Res. 27: 722-736.

8. Hunt M., Silva ND, Otto TD, Parkhill J, Keane JA., Harris SR. 2015. Circlator: automated circularization of genome assemblies using long sequencing reads. Genome Biol. 16: 294.

9. Aziz RK., Bartels D, Best AA, DeJongh M, Disz T, Edwards RA, et al. 2008. The RAST Server: rapid annotations using subsystems technology. BMC Genomics 9: 75. doi: 10.1186/ 1471-2164-9-75.

10. Lowe, TM., Eddy, SR. 1997. tRNAscan-SE: a program for improved detection of transfer RNA genes in genomic sequence. Nucleic Acids Res. 25: 955-964.

11. Lagesen K, Hallin P, Rodland EA, Staerfeldt HH, Rognes T, Ussery DW. 2007. RNAmmer: consistent and rapid annotation of ribosomal RNA genes. Nucleic Acids Res. 35: 3100-3108.

12. Krzywinski M, Schein J, Birol I, Connors J, Gascoyne R, Horsman D, et al. 2009. Circos: an information aesthetic for comparative genomics. Genome Res. 19: 1639-1645.

13. Darling AE, Mau B, Blattner FR, Perna NT. 2004. Mauve: Multiple alignment of conserved genomic sequence with rearrangements. Genome Res. 14: 1394-1403.

14. Pritchard L. 2017. pyani: Python module for average nucleotide identity analyses. Available from https://github.com/ widdowquinn/pyani.

15. Darling AE, Mau B, Perna NT. 2010. ProgressiveMauve: multiple genome alignment with gene gain, loss and rearrangement. PLoS One 5: 11147.

16. Seemann T. 2014. Prokka: rapid prokaryotic genome annotation. Bioinformatics 30: 2068-2069.

17. Page AJ, Cummins CA, Hunt M, Wong VK, Reuter S, Holden MT, et al. 2015. Roary: Rapid large-scale prokaryote pan genome analysis. Bioinformatics 31: 3691-3693.

18. Conway JR., Lex A, Gehlenborg N. 2017. UpSetR: an R package for the visualization of intersecting sets and their properties. Bioinformatics 33: 2938-2940.

19. Karp PD, Paley S, Romero P. 2002. The Pathway Tools software. Bioinformatics 18: S225-S232.

20. Saitou N, Nei M. 1987. The neighbor-joining method: a new method for reconstructing phylogenetic trees. Mol. Biol. Evol. 4: 406-425.

21. Tatusov, RL., Galperin, MY., Natale, DA., Koonin, EV. 2000. The COG database: a tool for genome-scale analysis of protein functions and evolution. Nucleic Acids Res. 28: 33-36.
22. De Maayer P, Anderson D, Cary C, Cowan DA. 2014. Some like it cold: understanding the survival strategies of psychrophiles. EMBO Rep. 15: 508-5017.

23. Rodrigues DF, Tiedje JM. 2008. Coping with our cold planet. Appl. Environ. Microbiol. 74: 1677-1686.

24. Zhang S, Song W, Yu M, Lin X. 2017. Comparative genomics analysis of five Psychrobacter strains isolated from worldwide habitats reveal high intra-genus variations. Extremophiles 21: 581-589.

25. Math RK, Jin HM, Kim JM, Hahn Y, Park W, Madsen EL, et al. 2012. Comparative genomics reveals adaptation by Alteromonas sp. SN2 to marine tidal-flat conditions: cold tolerance and aromatic hydrocarbon metabolism. PLoS One 7: e35784.

26. Du MZ, Wen W, Qin L, Liu S, Zhang A, Zhang Y, et al. 2017. Co-adaption of tRNA gene copy number and amino acid usage influences translation rates in three life domains. DNA Res. 24: 623-633.

27. McDonald MJ, Chou CH, Swamy KB, Huang HD, Leu J. 2015. The evolutionary dynamics of tRNA-gene copy number and codon-use in E. coli. BMC Evol. Biol. 15: 163.

28. Dethlefsen L, Schmidt TM. 2007. Performance of the translational apparatus varies with the ecological strategies of bacteria. J. Bacteriol. 189: 3237-3245.

29. Kim M, Oh HS, Park SC, Chun J. 2014. Towards a taxonomic coherence between average nucleotide identity and $16 \mathrm{~S}$ rRNA gene sequence similarity for species demarcation of prokaryotes. Int. J. Syst. Evol. Microbiol. 64: 346-351.

30. Richter M, Rossello-Mora R. 2009. Shifting the genomic gold standard for the prokaryotic species definition. Proc. Natl. Acad. Sci. USA 106: 19126-19131.

31. Lapierre P, Gogarten JP. 2009. Estimating the size of the bacterial pan-genome. Trends Genetics 25: 107-110.

32. Vernikos G, Medini D, Riley DR., Tettelin H. 2015. Ten years of pan-genome analyses. Curr. Opin. Microbiol. 23: 148154.

33. Christel S, Fridlund J, Watkin EL, Dopson M. 2016. Acidithiobacillus ferrivorans SS3 presents little RNA transcript response related to cold stress during growth at $8^{\circ} \mathrm{C}$ suggesting it is a eurypsychrophile. Extremophiles 20: 903913.

34. Huynen MA, Dandekar T, Bork P. 1999. Variation and evolution of the citric-acid cycle: a genomic perspective. Trends Microbiol 7: 281-291.

35. Pitson SM, Mendz GL, Srinivasan S, Hazell SL. 1999. The tricarboxylic acid cycle of Helicobacter pylori. Eur. J. Biochem. 260: 258-267.

36. Kather B, Stingl K, van der Rest ME, Altendorf K, Molenaar D. 2000. Another unusual type of citric acid cycle enzyme in Helicobacter pylori: the malate:quinone oxidoreductase. J. Bacteriol. 182: 3204-3209. 